\title{
Gathering of seven autonomous mobile robots on triangular grids
}

\author{
Masahiro Shibata \\ Graduate School of Computer Science and Systems Engineering \\ Kyushu Institute of Technology \\ Fukuoka, Japan \\ shibata@cse.kyutech.ac.jp \\ Yuichi Sudo \\ Graduate School of Information Science and Technology \\ Osaka University \\ Osaka, Japan \\ y-sudou@ist.osaka-u.ac.jp \\ Yonghwan Kim \\ Graduate School of Computer Science and Engineering \\ Nagoya Institute of Technology \\ Aichi, Japan \\ kim@nitech.ac.jp
}

\author{
Masaki Ohyabu \\ Graduate School of Computer Science and Engineering \\ Nagoya Institute of Technology \\ Aichi, Japan \\ oyabu@moss.elcom.nitech.ac.jp
}

\author{
Junya Nakamura \\ Information and Media Center \\ Toyohashi University of Technology \\ Aichi, Japan \\ junya@imc.tut.ac.jp
}

\begin{abstract}
In this paper, we consider the gathering problem of seven autonomous mobile robots on triangular grids. The gathering problem requires that, starting from any connected initial configuration where a subgraph induced by all robot nodes (nodes where a robot exists) constitutes one connected graph, robots reach a configuration such that the maximum distance between two robots is minimized. For the case of seven robots, gathering is achieved when one robot has six adjacent robot nodes (they form a shape like a hexagon). In this paper, we aim to clarify the relationship between the capability of robots and the solvability of gathering on a triangular grid. In particular, we focus on visibility range of robots. To discuss the solvability of the problem in terms of the visibility range, we consider strong assumptions except for visibility range. Concretely, we assume that robots are fully synchronous and they agree on the direction and orientation of the $x$-axis, and chirality in the triangular grid. In this setting, we first consider the weakest assumption about visibility range, i.e., robots with visibility range 1 . In this case, we show that there exists no collision-free algorithm to solve the gathering problem. Next, we extend the visibility range to 2 . In this case, we show that our algorithm can solve the problem from any connected initial configuration. Thus, the proposed algorithm is optimal in terms of visibility range.

Index Terms-distributed system, mobile robot, gathering problem, triangular grid
\end{abstract}

\section{INTRODUCTION}

\section{A. Background}

Studies for (autonomous) mobile robot systems have emerged recently in the field of Distributed Computing. Robots aim to achieve some tasks with limited capabilities. Most studies assume that robots are uniform (they execute the same algorithm and cannot be distinguished by their appearance) and oblivious (they cannot remember their past actions). In addition, it is assumed that robots cannot communicate with other robots explicitly. Instead, the communication is done implicitly; each robot can observe the positions of the other robots.

\section{B. Related work}

Since Suzuki and Yamashita presented the pioneering work [1], many problems have been studied. For example, the gathering problem, which requires all robots to meet at a non-predetermined single point, has been studied in various environments. In the two-dimensional Euclidean space (a.k.a., the continuous model), Suzuki and Yamashita [1] showed that when robots are not fully synchronous, the deterministic gathering of two robots is impossible without additional assumptions. This impossibility result was generalized to an even number of robots initially located evenly at two positions by Courtieu et al. [2]. By contrast, Dieudonné and Petit [3] showed that, by adding the assumption that robots can count the exact number of robots at each position, an odd number of robots can gather from any initial position.

The gathering problem in the discrete space (a.k.a., the graph model) has also been studied. In the discrete space, robots stay at fixed positions (the nodes of the graph), and move from one position to the next position through edges of the graph. For (square) grid graphs, D'Angelo et al. [4] and Castenow et al. [5] proposed algorithms to solve the gathering problem. For ring graphs, Klasing et al. [6], [7] showed the 
existence of unsolvable initial configurations and proposed algorithms to solve the problem from some specific initial configurations. D'Angelo et al. [8] proposed an algorithm to solve the problem from any solvable initial configuration. Stefano and Navarra [9] analyzed the required total number of robot moves to solve the gathering problem in rings.

As a variant of mobile robots, gathering of fat robots is considered $[10]-[12]$. Each fat robot dominates a space of a unit disc. There are several definitions of the gathering problem for fat robots, e.g., robots achieve gathering when (i) they form a connected configuration (each robot touches at least one other robot and all robots form one connected formation) or (ii) they reach a configuration such that the maximum distance between two robots is minimized. For both the definitions, a collision is not allowed. Thus, introducing sizes gives several definitions of the gathering problem, which is an interesting point. Czyzowicz et al. [10] considered gathering of (i) for three or four fat robots in the continuous model, and Chrysovalandis et al. [11] studied gathering of (i) for arbitrary number of robots. Ito et al. [12] considered gathering of (ii) on discrete square grids.

Recently, one of computational models for programmable matter, amoebot has been introduced [13]. Each amoebot moves on a triangular grid and occupies one or two adjacent nodes. Each amoebot has a finite memory, limited visibility range, and ability to communicate with a robot staying at an adjacent node. Several problems using amoebots have been considered, such as leader election [14], gathering [15], and shape formation (or pattern formation) [16], [17]. Recall that while amoebots have finite memory and communication capability, (standard) autonomous mobile robots have no memory or communication capability. Hence, the mobile robot model is weaker than the amoebot model, and it is interesting to clarify solvability of problems between the mobile robot model and the amoebot model.

Meanwhile, when considering a discrete space, a space filled by regular polygons is sometimes preferable because its simple structure helps to design an algorithm and to discuss the solvability of a problem among various robot models. In addition, (i) only triangular, square, and hexagonal grids are discrete spaces filled by regular polygons, (ii) gathering on a square gird has already been studied [12], and (iii) recently the amoebot model has been extensively studied on a triangular grid. Hence, in this paper we consider gathering of mobile robots on a triangular grid.

\section{Our contribution}

In this paper, we consider the gathering problem of seven mobile robots on triangular grids. We say in this paper that gathering is achieved when robots reach a configuration such that the maximum distance between two robots is minimized. For the case of seven robots, letting a robot node be a node where a robot exists, gathering is achieved when one robot has six adjacent robot nodes like Fig.1. This implies that robots form a (filled) hexagon. In this paper, we aim to clarify the relationship between the capability of robots and

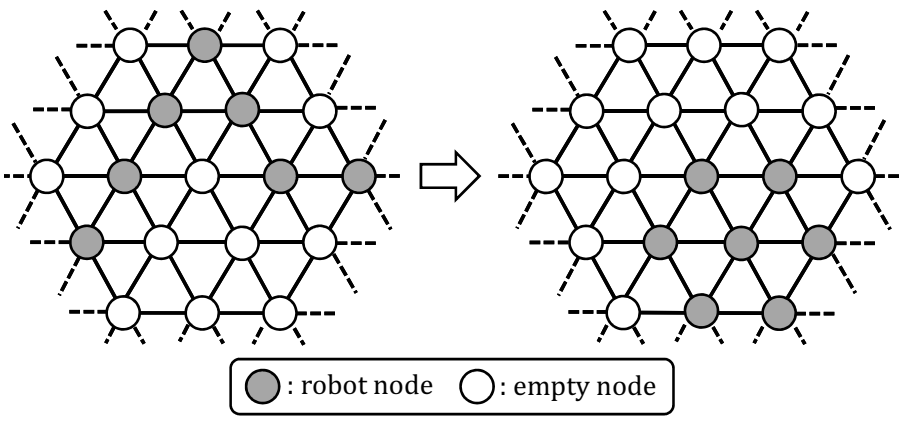

Fig. 1. An example of the gathering problem.

the solvability of the gathering problem on a triangular grid. In particular, we focus on visibility range of robots. To discuss the solvability of the problem in terms of the visibility range, we consider strong assumptions except for visibility range. Concretely, we assume that robots are fully synchronous, and they agree on the direction and orientation of the $x$-axis, and chirality in the triangular grid. In this setting, we first consider the weakest assumption about visibility range, i.e., robots with visibility range 1 . In this case, we show that there exists no collision-free algorithm to solve the gathering problem. Next, we extend the visibility range to 2 . In this case, we show that our algorithm can solve the problem from any connected initial configuration. Thus, the proposed algorithm is optimal in terms of visibility range.

\section{Preliminaries}

\section{A. System model}

An (infinite) triangular grid is an undirected graph $G=$ $(V, E)$, where $V$ is the set of nodes and $E$ is the set of edges. The grid has one special node called origin, and we denote it by $v_{o}$. Each node $v_{j} \in V$ has six adjacent nodes: east $\left(v_{E}^{j}\right.$ or E), southeast $\left(v_{S E}^{j}\right.$ or $\left.\mathrm{SE}\right)$, southwest $\left(v_{S W}^{j}\right.$ or $\left.\mathrm{SW}\right)$, west $\left(v_{W}^{j}\right.$ or $\mathrm{W})$, northwest $\left(v_{N W}^{j}\right.$ or NW), and northeast $\left(v_{N E}^{j}\right.$ or NE). The axis including $v_{o}$ and $v_{E}^{o}$ (resp., $v_{o}$ and $v_{N E}^{o}$ ) is called the $x$ axis (resp., y-axis) 1 An example is given in Fig.2. In addition, a sequence of $k+1$ distinct nodes $\left(v_{0}, v_{1}, \ldots, v_{k}\right)$ is called a path with length $k$ if $\left\{v_{i}, v_{i+1}\right\} \in E$ for all $i \in[0, k-1]$. The distance between two nodes is defined as the length of the shortest path between them.

In this paper, we consider seven mobile robots and denote the robot set by $R=\left\{r_{0}, r_{1}, \ldots, r_{6}\right\}$. Robots considered here have the following characteristics. Robots are uniform, that is, they execute the same algorithm and cannot be distinguished by their appearance. Robots are oblivious, that is, they have no persistent memory and cannot remember their past actions. Robots cannot communicate with other robots directly. However, robots have limited visibility range and they can observe the positions of other robots within the range. This means that robots can communicate implicitly by their positions.

\footnotetext{
${ }^{1}$ Although the origin, the $x$-axis, and the $y$-axis are terms of the coordinate system, we use these terms for explanation. In the following, we use several terms of the coordinate system.
} 


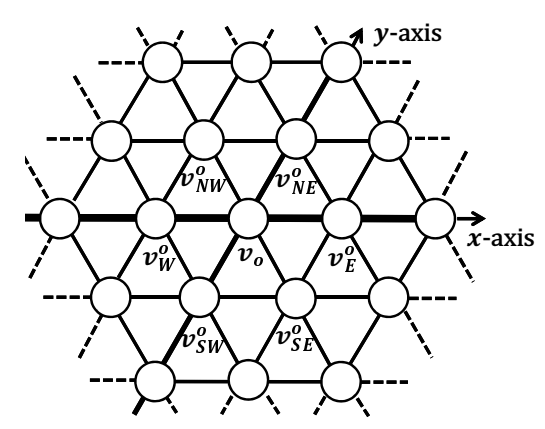

Fig. 2. An example of a triangular grid.

We consider two problem settings about robots: robots with visibility range 1 and robots with visibility range 2 . Robots with visibility range 1 can observe nodes within distance 1 , that is, they can only observe their six adjacent nodes. On the other hand, robots with visibility range 2 can observe nodes within distance 2 (eighteen nodes in total). We assume that they are transparent, that is, even if a robot $r_{i}$ and several robots exist on the same axis, $r_{i}$ can observe all the robots on the axis within its visibility range. Robots do not know the position of the origin, but they agree on the direction and orientation of the $x$-axis, and chirality (the orientation of axes, e.g., clockwise or counter-clockwise) in the triangular gird.

Each robot executes the algorithm by repeating LookCompute-Move cycles. At the beginning of each cycle, the robot observes positions of the other robots within its visibility range (Look phase). According to the observation, the robot computes whether it moves to its adjacent node or stays at the current node (Compute phase). If the robot decides to move, it moves to the node by the end of the cycle (Move phase). Robots are fully synchronous (FSYNC), that is, all robots start every cycle simultaneously and execute each phase synchronously. We assume that a collision is not allowed during execution of the algorithm. Here, a collision represents a situation such that two robots traverse the same edge from different directions or several robots exist at the same node. Concretely, the following three behaviors are not allowed: (a) some robot $r_{i}$ (resp., $r_{j}$ ) staying at node $v_{p}$ (resp., $v_{q}$ ) moves to $v_{q}$ (resp., $v_{p}$ ), (b) some robot $r_{i}$ staying at node $v_{p}$ remains at $v_{p}$ and robot $r_{j}$ staying at node $v_{q}$ moves to $v_{p}$, and (c) several robots move to the same empty node.

A configuration of the system is defined as the set of locations of each robot. Here, the location of a robot $r_{i}$ is defined as the position that (1) $r_{i}$ is currently staying at and (2) is represented as an intersection of an axis parallel to the $x$-axis and an axis parallel to the $y$-axis. Each axis $a x$ is represented by (i) whether it is parallel to the $x$-axis or the $y$-axis and which direction it is far from the axis, and (ii) the number of axes between the axis including $v_{o}$ (i.e., the $x$-axis or the $y$-axis) and ax. However, robots do not know the position of $v_{o}$ and they cannot use information of (global) locations. A node is called a robot node if the node is occupied by a robot. Otherwise, the node is called an empty

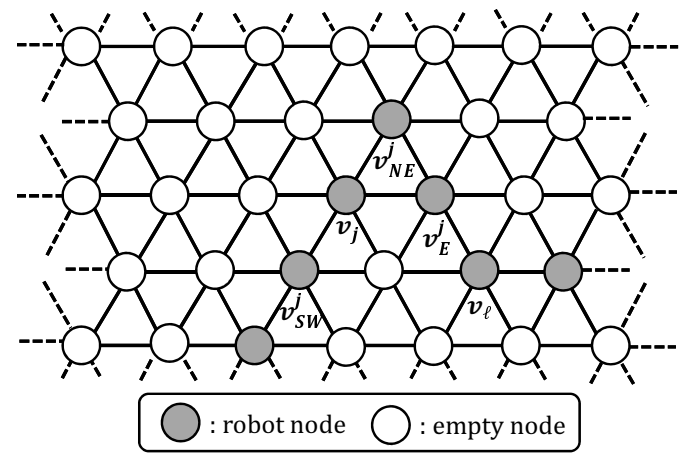

Fig. 3. An example of a configuration.

node. We assume that the initial configuration is connected, that is, the subgraph of $G$ induced by the seven robot nodes is connected. This assumption of connectivity is necessary because, if a configuration becomes unconnected and a robot $r$ has no adjacent robot node, $r$ cannot know the direction to reconstruct a connected configuration due to obliviousness, which implies that robots cannot achieve gathering.

When a robot executes a Look phase, it gets a view of the system. A view of a robot is defined as the set of robot nodes within its visibility range. For example, in Fig. 3, a robot at node $v_{j}$ recognizes that nodes $v_{E}^{j}, v_{S W}^{j}$, and $v_{N E}^{j}$ are robot nodes when its visibility range is 1 and recognizes that nodes $v_{k}$ and $v_{\ell}$ are also robot nodes when its visibility range is 2 .

\section{B. Gathering problem}

The gathering problem of mobile robots requires that starting from any connected initial configuration, the robots terminate in a configuration such that the maximum distance between two robot nodes is minimized. In the case of seven robots, gathering is achieved when one robot has six adjacent robot nodes (Fig.11). Concretely, we define the problem as follows.

Definition 1. A collision-free algorithm $\mathcal{A}$ solves the gathering problem of seven autonomous mobile robots on a triangular grid if and only if the system reaches a configuration such that one robot has six adjacent robot nodes and no robot moves thereafter, without a collision throughout the execution of $\mathcal{A}$,

\section{RoBOtS WITH VISIBILITY RANGE 1}

In this section, for robot with visibility range 1 , we show that there exists no collision-free algorithm to solve the problem.

Theorem 1. For robots with visibility range 1, there exists no collision-free algorithm to solve the gathering problem even in the fully synchronous (FSYNC) model.

Proof. We show the proof by contradiction, that is, we assume that there exists a collision-free algorithm $\mathcal{A}$ to solve the gathering problem from any connected initial configuration. In the proof, we consider several configurations and robot behaviors, and show that if some robot moves to some direction by 


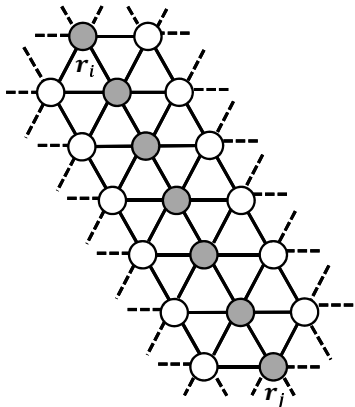

(a)

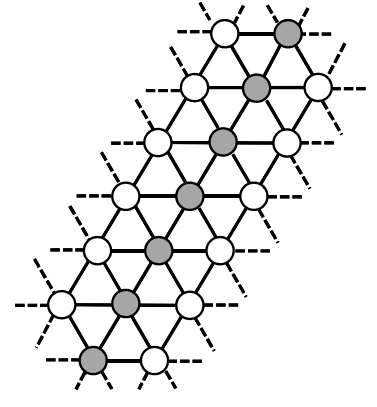

(b)
Fig. 4. Configurations we consider in the proof.

Algorithm $\mathcal{A}$, several robots cannot move anywhere (i.e., they have to stay at the current nodes) since a collision occurs or the configuration becomes unconnected. Eventually, we show that there is a configuration such that all robots need to stay at the current nodes and they cannot achieve gathering, which is a contradiction.

First, we consider the configuration of Fig.4 (a). In the figure, robot $r_{i}$ (resp., $r_{j}$ ) has one adjacent robot node SE (resp., NW) and the other robots have two adjacent robot nodes SE and NW, respectively. In such a configuration, we first show that intermediate robots cannot leave the current nodes.

Lemma 1. A robot with two adjacent robot nodes $W$ and $E$, $S W$ and NE, or NW and SE must stay at the current node.

Proof. We consider configurations of Fig.5 In each configuration, robots $r_{i}$ and $r_{j}$ have two adjacent robot nodes $\mathrm{W}$ and $\mathrm{E}$. On the other hand, robots $r_{p}$ and $r_{q}$ have three adjacent robot nodes and they must stay at the current nodes because they cannot detect whether the current configuration is a gatheringachieved configuration or not. In addition, if $r_{i}$ moves to $\mathrm{W}$, $\mathrm{NW}$, or $\mathrm{SW}, r_{j}$ also moves to the same direction because they have the same view. Then, either in Fig.5 (a) or (b), wherever $r_{k}$ moves to, a collision occurs or the configuration becomes unconnected. By a similar discussion, when $r_{i}$ and $r_{j}$ move to $\mathrm{E}, \mathrm{NE}$, or $\mathrm{SE}$, it causes a collision or an unconnected configuration either in Fig.5 (c) or (d). Thus, a robot with two adjacent robot nodes $\mathrm{E}$ and $\mathrm{W}$ cannot leave the current node. By the similar discussion, we can show that a robot with two adjacent robot nodes SW and NE, or NW and SE must stay at the current node. Thus, the lemma follows.

By this lemma, we can have the following two colloraries.

Collorary 1. A robot with one adjacent robot node E, SE, SW, $W, N W$, or $N E$ can move only to $N E$ or $S E, E$ or $S W, S E$ or $W, S W$ or NW, W or NE, or NW or E if it moves, respectively.

Collorary 2. A robot with two adjacent robot nodes $E$ and $S W, S E$ and $W, S W$ and $N W, W$ and $N E, N W$ and $E$, or $N E$ and $S E$ can move only to node SE, SW, W, NW, NE, or E if it moves, respectively.

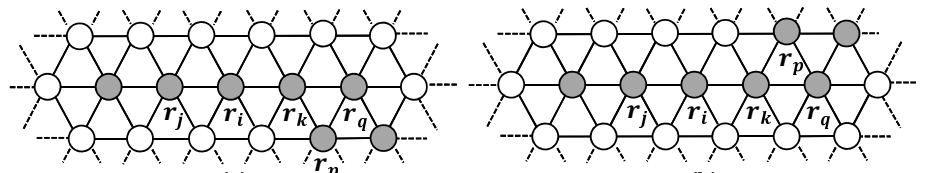

(a)

(b)

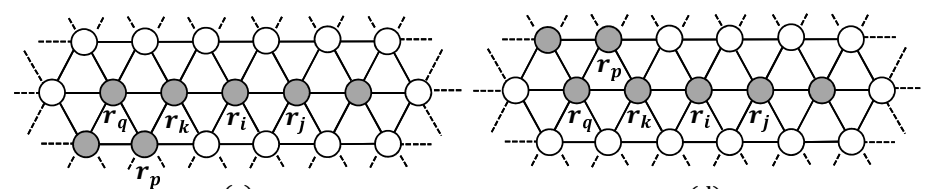

(c)

(d)

Fig. 5. An example of a configuration that a robot with two adjacent robot nodes $\mathrm{W}$ and $\mathrm{E}$ must stay at the current node.

By Lemma 1, intermediate robots in Fig.4 (a) cannot leave the current nodes, and hence $r_{i}$ or $r_{j}$ has to leave the current node. Without loss of generality, we assume that in $\mathcal{A}$ robot $r_{i}$ with one adjacent robot node SE moves to SW. Notice that $r_{i}$ can move only to SW or E by Collorary 1 . In the following, we consider several robot behaviors and eventually show that a robot with one adjacent robot node NE or SW must stay at the current node. Then, in a configuration of Fig.4 (b), all robots must stay at the current nodes and they cannot solve the gathering problem, which is a contradiction.

When a robot with one adjacent robot node SE moves to SW, several robot behaviors are not allowed since a collision occurs, as shown in Fig.6(for simplicity, we omit robot nodes unrelated to prohibited robot behaviors). Concretely, we have the following proposition.

Proposition 1. When a robot with one adjacent robot node $S E$ moves to $S W$, the following four robot behaviors are not allowed: (a) a robot with one adjacent robot node NE moves to $N W$, (b) a robot with two adjacent robot nodes NW and $S W$ moves to $W$, (c) a robot with one adjacent robot node $E$ moves to $N E$, and $(d)$ a robot with two adjacent robot nodes $N W$ and E moves to $N E$.

In the following, we consider the following five cases: (1) a robot with one adjacent robot node NW moves to W, (2) a robot with one adjacent robot node SW moves to SE, (3) a robot with one adjacent robot node NE moves to E, (4) a robot with one adjacent robot node NW moves to NE, and (5) a robot with one adjacent robot node SW moves to $\mathrm{W}$. In each case, we show that the assumed robot behavior is not allowed. Thus, by Proposition 1 1 (a) and cases (2), (3), and (5), robots cannot achieve gathering from the configuration of Fig. 4 (b), which is a contradiction (results of cases (1) and (4) are used for cases (2), (3), and (5)).

Case 1: a robot with one adjacent robot node NW moves to W. In this case, as shown in Fig.7, the following three robot behaviors are not allowed: (a) a robot with two adjacent robot nodes W and SE moves to SW, (b) a robot with one adjacent robot node $\mathrm{E}$ moves to $\mathrm{SE}$, and (c) a robot with one adjacent robot node NE moves to $\mathrm{E}$. Then, let us consider the configuration of Fig. 8 In the configuration, by Proposition 1 


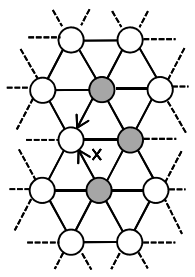

(a)

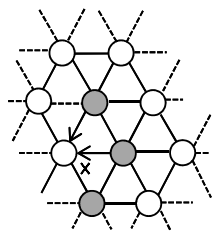

(b)

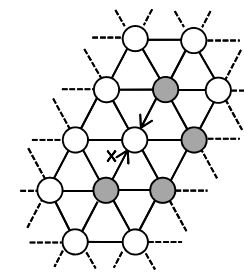

(c)

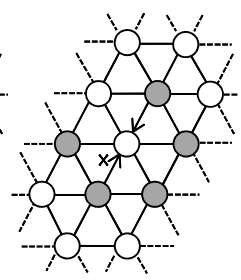

(d)

Fig. 6. Prohibited behaviors when a robot with one adjacent robot node SE moves to SW.

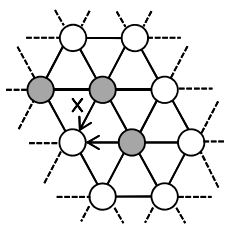

(a)

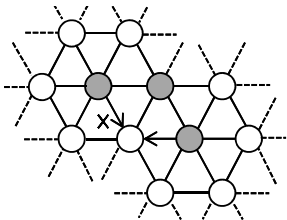

(b)

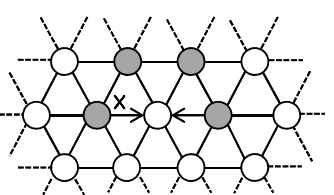

(c)

Fig. 7. Prohibited behaviors when a robot with one adjacent robot node NW moves to node $\mathrm{W}$.

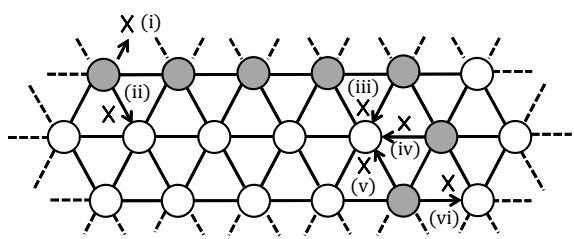

Fig. 8. An unsolvable configuration when a robot with one adjacent robot node NW moves to W ((i): by Fig.6(c), (ii): by Fig.7(b), (iii): by Fig.7(a), (iv): by Fig.6(b), (v): by Fig.6(a), (vi): by Fig.7(c)).

and the above discussion, no robot can leave the current node and robots cannot achieve gathering, which is a contradiction. Thus, we have the following lemma.

Lemma 2. A robot with one adjacent robot node NW cannot move to node $W$.

Case 2: a robot with one adjacent robot node SW moves to SE. In this case, as shown in Fig.9, the following four robot behaviors are not allowed: (a) a robot with one adjacent robot node NW moves to NE, (b) a robot with two adjacent robot nodes NE and SE moves to E, (c) a robot with one adjacent robot node $\mathrm{W}$ moves to $\mathrm{NW}$, and (d) a robot with two adjacent robot nodes NW and E (resp., W and NE) moves to NE (resp., NW). Then, in a configuration of Fig.10, only robot $r_{p}$ with two adjacent robot nodes SW and $\mathrm{E}$ can move to SE or robot $r_{q}$ with two adjacent robot nodes $\mathrm{W}$ and SE can move to SW by the above discussion and Lemmas 1 and 2 and Collorary 2 . We consider each of the behaviors and show for both the cases that robots cannot achieve gathering from some configuration.

Case 2-1: robot $r_{p}$ moves to $S E$. In this case, clearly a robot with one adjacent robot node NE cannot move to $\mathrm{E}$ and a robot with one adjacent robot node $\mathrm{W}$ cannot move to SW since a collision occurs. In addition, when considering a configuration of Fig 11 (a), only robot $r_{i}$ with one adjacent robot node $\mathrm{E}$

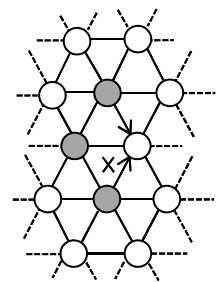

(a)

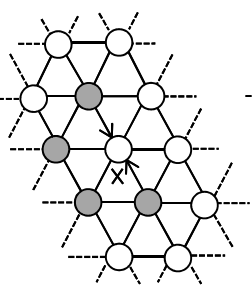

(c)

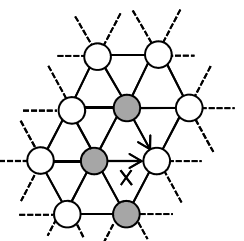

(b)

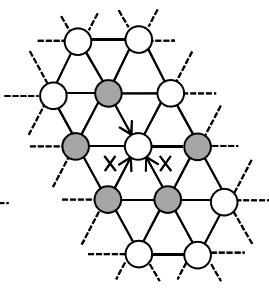

(d)

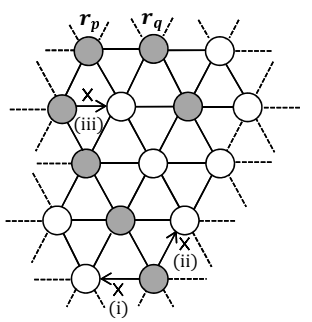

Fig. 10. A configuration such that only $r_{p}$ or $r_{q}$ can leave the current node ((i): by Lemma 2 (ii): by Fig. 9 (a), (iii): by Fig. 9 (b)).

Fig. 9. Prohibited behaviors when a robot with one adjacent robot node SW moves to SE.

can leave the current node and it needs to move to SE by the previous discussions. Now, we consider the configuration of Fig. 12 (a). In the figure, robot $r_{1}, r_{3}$, and $r_{5}$ move to SE and the other robots must stay at the current nodes. Then, the system reaches the configuration of Fig.12 (b). In the configuration, robots $r_{0}, r_{2}, r_{4}$, and $r_{6}$ move to $\mathrm{SE}$ and the other robots must stay at the current nodes. Then, the system reaches the configuration of Fig.12 (a). Thus, robots repeat configurations of Fig.12 (a) and (b) forever and they cannot achieve gathering, which is a contradiction.

Case 2-2: robot $r_{q}$ moves to $S W$. In this case, clearly a robot with one adjacent robot node $\mathrm{E}$ cannot move to SE since a collision occurs. In addition, when considering a configuration of Fig.11 (b), only robot $r_{i}$ with one adjacent robot node W can leave the current node and it needs to move to SW by the previous discussions. Now, we consider the configuration of Fig.13 (a). In the figure, robot $r_{0}, r_{2}, r_{4}$, and $r_{6}$ move to SW and the other robots must stay at the current nodes. Then, the system reaches the configuration of Fig.13 (b). In the configuration, robots $r_{1}, r_{3}$, and $r_{5}$ move to $\mathrm{SW}$ and the other robots must stay at the current nodes. Then, the system reaches the configuration of Fig.13 (a). Thus, robots repeat configurations of Fig.13 (a) and (b) forever and they cannot achieve gathering, which is a contradiction. Thus, we have the following lemma.

Lemma 3. A robot with one adjacent robot node SW cannot move to node SE.

Case 3: a robot with one adjacent robot node NE moves to E. In this case, the following two robot behaviors are not allowed (Fig.14): a robot with two adjacent robot nodes SW and $\mathrm{E}$ (resp., W and SE) moves to SE (resp., SW). Then, in a configuration of Fig. 15, it is necessary that at least a robot $r_{p}$ with two adjacent robot nodes NE and SE moves to $\mathrm{E}$ or a robot $r_{q}$ with one adjacent robot node with NW 


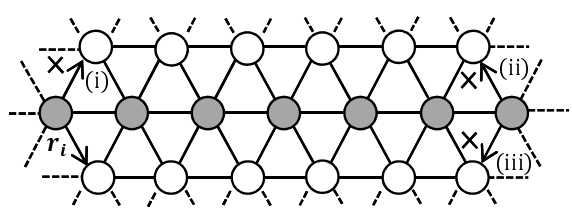

(a)

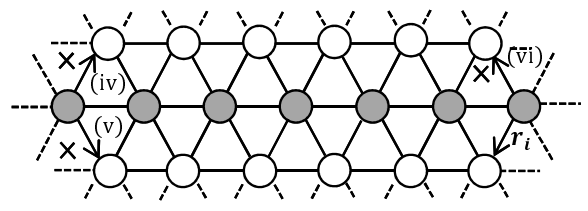

(b)

Fig. 11. (a): An example such that only a robot $r_{i}$ with one adjacent robot node $\mathrm{E}$ can leave the current node in Case 2-1 ((i): by Fig. 6 (c), (ii): by Fig.9 (c), (iii): prohibited behavior when a robot with two adjacent robot nodes SW and E moves to SE), (b): An example such that only a robot $r_{i}$ with one adjacent robot node $\mathrm{W}$ can leave the current node in Case 2-2 ((iv): by Fig. 6(c), (v): prohibited behavior when a robot with two adjacent robot nodes W and SE moves to SW, (vi): by Fig.9(c)).

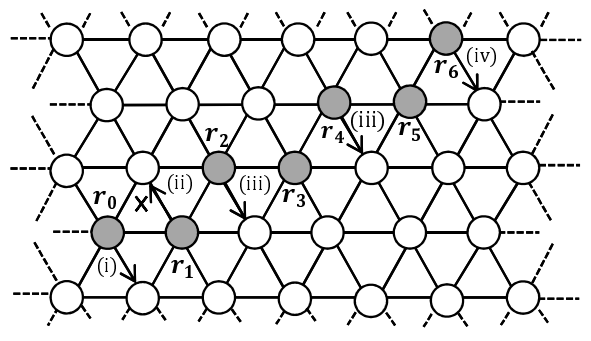

(a)

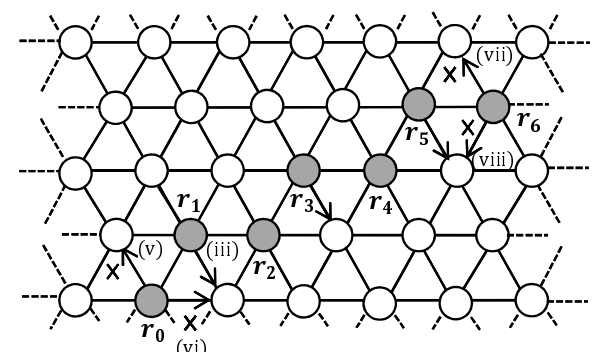

(b)

Fig. 12. Configurations that robots repeat alternately ((i): by Fig.11(a), (ii): by Fig.9 (d), (iii): assumption of Case 2-1, (iv): assumption of Case 2, (v): by Fig.6 (a), (vi): prohibited behavior when a robot with two adjacent robot nodes SW and E moves to SE, (vii): by Fig.9 (c), (viii): prohibited behavior when a robot with two adjacent robot nodes SW and E moves to SE).

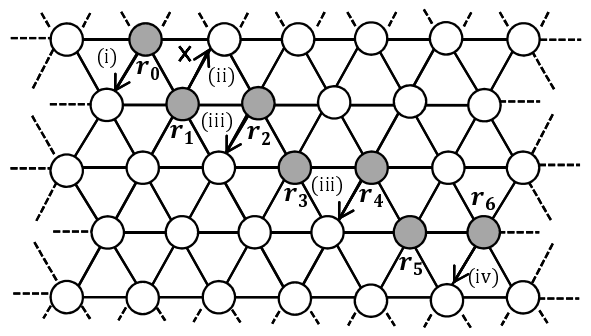

(a)

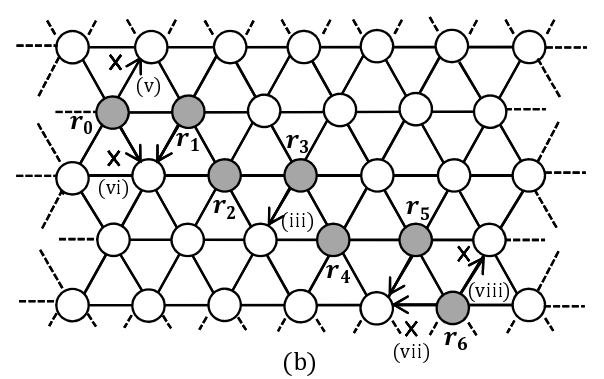

(b)

Fig. 13. Configurations that robots repeat alternately ((i): assumption of Algorithm $\mathcal{A}$, (ii): by Fig.9(d), (iii): assumption of Case 2-2, (iv): by Fig.11 (b), (v): by Fig.6 (c), (vi): prohibited behavior when a robot with two adjacent robot nodes W and SE moves to SW, (vii): by Lemma 2 (viii): by Fig.9 (a)).

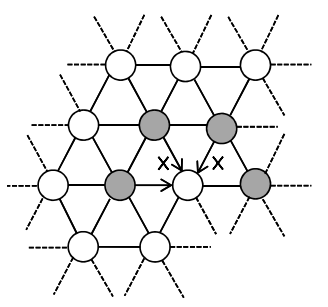

Fig. 14. Prohibited behaviors when a robot with one adjacent robot node NE moves to E.

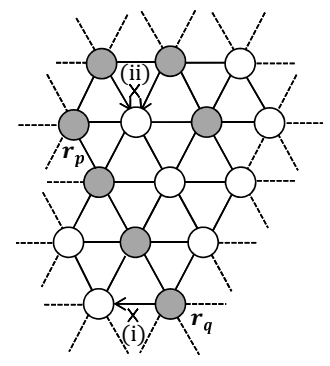

Fig. 15. A configuration such that only $r_{p}$ or $r_{p}$ can leave the current node ((i): by Lemma 2 (ii): by Fig. 14.

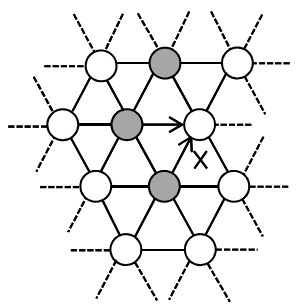

(a)

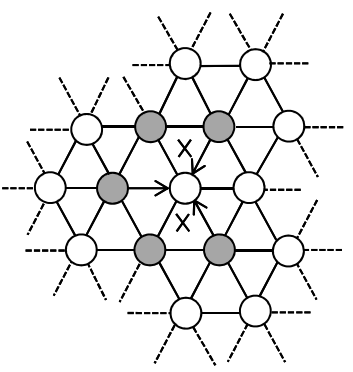

(b)
Fig. 16. Prohibited behaviors when a robot with two adjacent robot node NE and SE moves to E.

two robot behaviors are not allowed (Fig. 16): (a) a robot with one adjacent robot node NW moves to NE and (b) a robot with one adjacent robot node $\mathrm{W}$ moves to $\mathrm{NW}$ or $\mathrm{SW}$. In moves to NE by the previous discussions. We consider each of the behaviors and show for both the cases that robots cannot achieve gathering from some configuration.

Case 3-1: robot $r_{p}$ moves to $E$. In this case, the following 


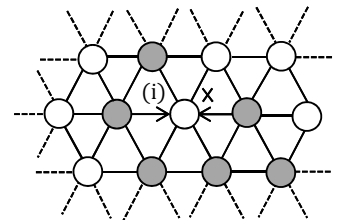

(a)

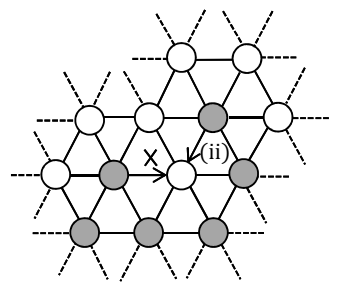

(b)
Fig. 17. Configurations showing that a robot with two adjacent robot nodes SW and SE must stay at the current node ((i): assumption of Case 3-1, (ii): assumption of Algorithm $\mathcal{A}$ ).

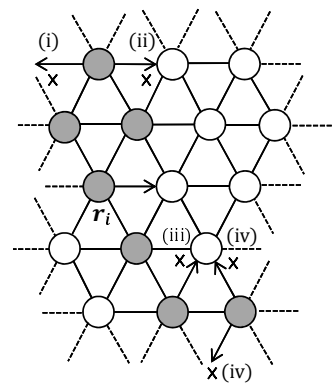

Fig. 18. A configuration such that only robot $r_{i}$ with three adjacent robot nodes NW, NE, and SE can leave the current node ((i): by Fig.17 (a), (ii): by Fig.17.(b), (iii): by Fig.6 (d), (iv): by Fig.16 (b)).

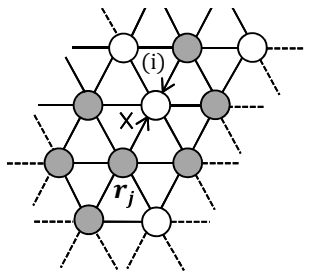

(a)

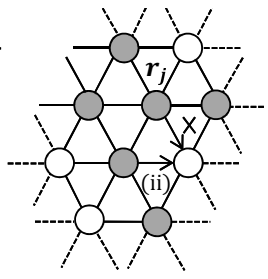

(b)
Fig. 19. Configurations showing that robot $r_{j}$ with four adjacent robot nodes E, SW, W, and NW must stay at the current node ((i): assumption of Algorithm $\mathcal{A}$, (ii): by Fig. 18 .

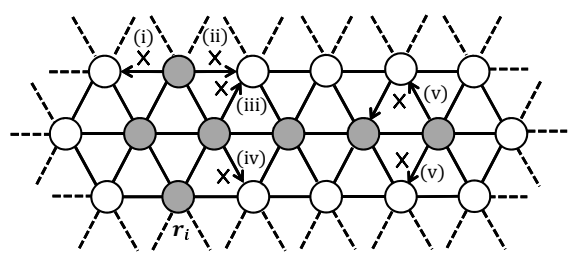

Fig. 20. A configuration such that only robot $r_{i}$ with two adjacent robot nodes NE and NW can leave the current node ((i): by Fig.17](a), (ii): by Fig.17 (b), (iii): by Fig.19 (a), (iv): by Fig.19 (b), (v): by Fig.16 (b)).

addition, when considering configurations of Fig.17, a robot with two adjacent robot node SW and SE must stay at the current node. Then, in a configuration of Fig. 18 only robot $r_{i}$ with three adjacent robot nodes NW, NE, and SE can leave the

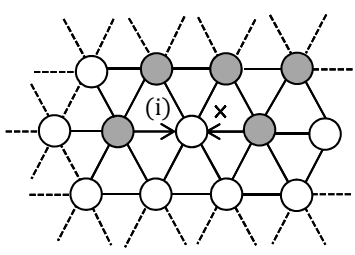

Fig. 21. An example showing that a robot with two adjacent robot nodes NE and NW cannot move to W ((i): assumption of Case 3).

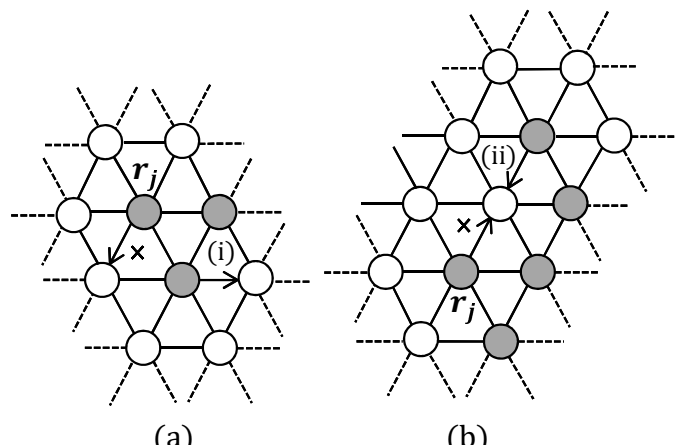

(a)

(b)

Fig. 22. Configurations showing that a robot with two adjacent robot nodes SW and SE must stay at the current node ((i): by Fig.21 (ii): assumption of Algorithm $\mathcal{A})$.

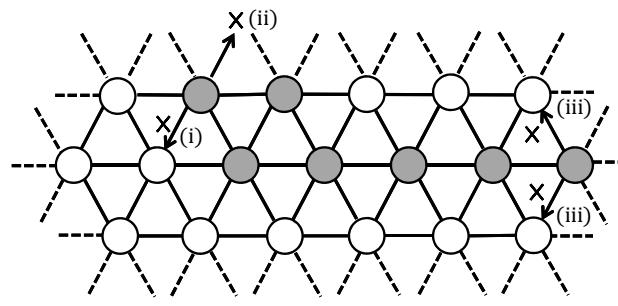

Fig. 23. An unsolvable configuration when a robot with two adjacent robot nodes NE and SE moves to E ((i): by Fig.22 (a), (ii): by Fig.22](b), (iii): by Fig.16(b)).

current node and it needs to move to $\mathrm{E}$ to avoid a collision or an unconnected configuration. In addition, when considering configurations of Fig. 19, a robot $r_{j}$ with four adjacent robots nodes E, SW, W, and NW must stay at the current node. Hence, in a configuration of Fig.20, only robot $r_{i}$ with two adjacent robot nodes NW and NE can leave the current node. Then, in a configuration of Fig.21, a collision occurs when $r_{i}$ moves to $\mathrm{W}$. Hence, it needs to move to E. Next, we consider the behavior of robot $r_{j}$ with two adjacent robot nodes $\mathrm{E}$ and SE. When it moves to SW, the configuration becomes unconnected if its southeast robot $r_{i}$ has two adjacent robot nodes NW and NE, and moves to E (Fig.22(a)). In addition, $r_{j}$ cannot move to NE since a collision occurs in a configuration of Fig. 22 (b). Thus, robot $r_{j}$ must stay at the current node. Finally, in a configuration of Fig.23 no robot can leave the current node and robots cannot achieve gathering. Therefore, a robot $r_{p}$ 


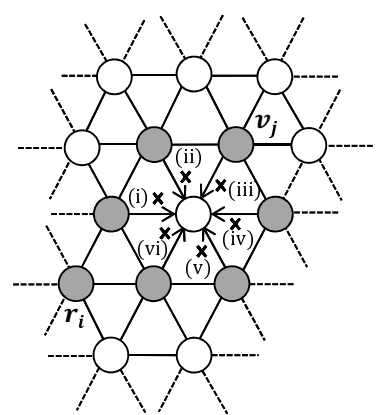

Fig. 24. A configuration such that only robot $r_{i}$ with two adjacent robot nodes NE and E can leave the current node ((i): by Case 3-1, (ii), (iii): by Fig.14 (iv)-(vi): behaviors that may cause a collision if a robot at $v_{j}$ has one adjacent robot node SE and moves to $\mathrm{SW}$ by the hypothesis of Algorithm $\mathcal{A}$ ).

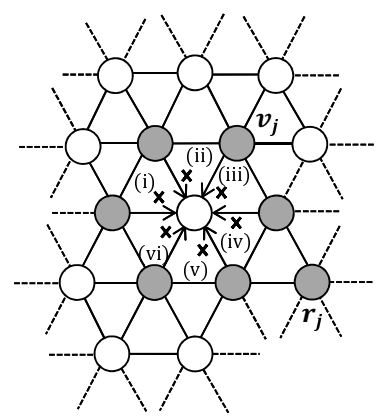

Fig. 26. A configuration such that only robot $r_{i}$ with two adjacent robot nodes NW and $\mathrm{W}$ can leave the current node ((i): by Case 3-1, (ii), (iii): by Fig. 14 (iv)-(vi): behaviors that may cause a collision if a robot at $v_{j}$ has one adjacent robot node SE and moves to SW by the hypothesis of Algorithm $\mathcal{A}$ ).

with two adjacent robot nodes NE and SE must stay at the current node. By a similar discussion, we can also show that a robot with three adjacent robot nodes NE, SE, and SW must stay at the current node.

Case 3-2: robot $r_{q}$ moves to $N E$. In this case, when considering a configuration of Fig. 24 only robot $r_{i}$ with two adjacent robot nodes $\mathrm{E}$ and $\mathrm{NE}$ can leave the current node. However, $r_{i}$ cannot move to $\mathrm{NW}$ since a collision occurs in a configuration of Fig.25 and it needs to move to SE. Similarly, in a configuration of Fig. 26 only robot $r_{j}$ with two adjacent robot nodes $\mathrm{NW}$ and $\mathrm{W}$ can leave the current node. However, $r_{j}$ cannot move to $\mathrm{SW}$ since a collision occurs in a configuration of Fig. 27 and it needs to move to NE. Now, we consider the configuration of Fig.28. In the figure, robot $r_{i}$ moves to $\mathrm{SE}$ and robot $r_{j}$ moves to $\mathrm{NE}$ by the above discussion. Then, the configuration become unconnected and robots cannot achieve gathering. Thus, a

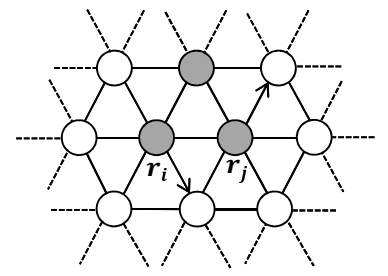

Fig. 28. A configuration that becomes unconnected after robots $r_{i}$ and $r_{j}$ move.
Fig. 29. Prohibited behaviors when a robot with one adjacent robot node NW moves to NE.

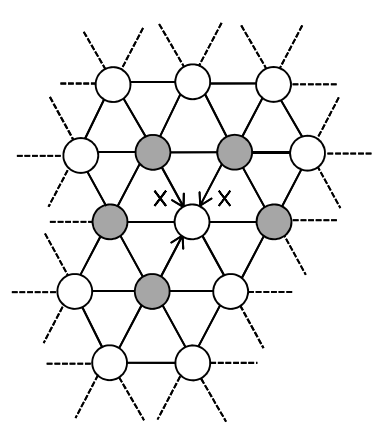

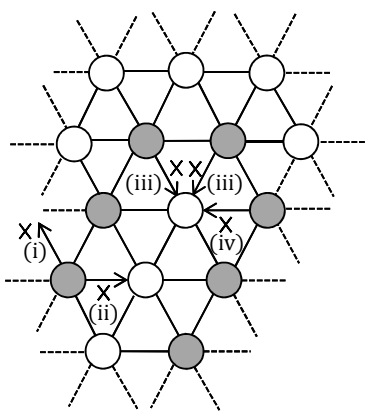

Fig. 30. A configuration such that no robot can leave the current node when a robot with one adjacent robot node NW moves to NE ((i): by Fig. 6 (a), (ii): by Lemma 4 (iii): by Fig. 29 (iv): by Fig.6(b)). robot $r_{q}$ with one adjacent robot node NW must stay at the current node. Therefore, robots cannot solve the problem from the configuration of Fig. 15 and we have the following lemma.

Lemma 4. A robot with one adjacent robot node NE cannot move to node $E$.

Case 4: a robot with one adjacent robot node NW moves to NE. In this case, the following two robot behaviors are not allowed (Fig.29): a robot with two adjacent robot nodes SW and $\mathrm{E}$ (resp., W and SE) moves to SE (resp., SW). Then, in a configuration of Fig. 30, no robot can leave the current node and robots cannot achieve gathering. Thus, we have the following lemma.

Lemma 5. A robot with one adjacent robot node NW cannot move to node NE.

Case 5: a robot with one adjacent robot node $S W$ moves to $\mathbf{W}$. In this case, the following four robot behaviors are not allowed (Fig. 31): (a): a robot with two adjacent robot nodes $\mathrm{NE}$ and SE moves to E, (b): a robot with two adjacent robot nodes NW and E moves to NE, (c): a robot with two adjacent robot nodes $\mathrm{W}$ and $\mathrm{NE}$ moves to $\mathrm{NW}$, and (d): a robot with three adjacent robot nodes NE, NW, and SE moves to E. Then, in a configuration of Fig. 32, it is necessary that robot $r_{p}$ with two adjacent robot nodes $\mathrm{SW}$ and $\mathrm{E}$ moves to SE or robot $r_{q}$ with two adjacent robot nodes $\mathrm{W}$ and SE moves to SW. We consider each of the behaviors and show for both the cases that robot cannot achieve gathering from some configuration. 


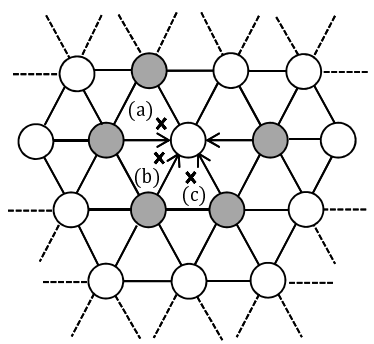

(i)

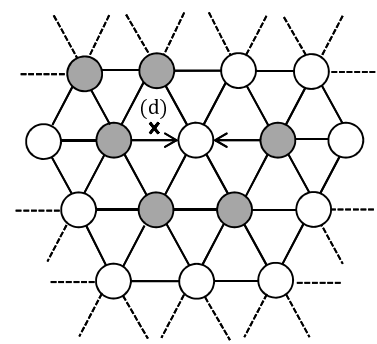

(ii)
Fig. 31. Prohibited behaviors when a robot with one adjacent robot node SW moves to $\mathrm{W}$.

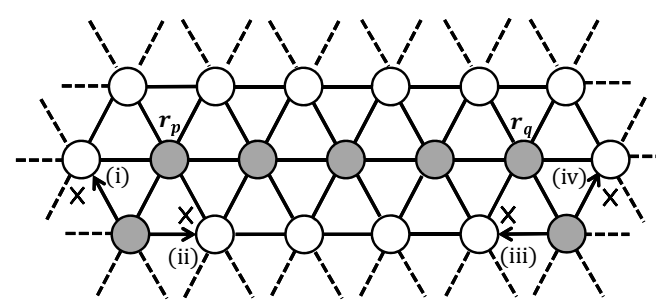

Fig. 32. A configuration such that only robot $r_{p}$ with two adjacent robot nodes SW and E or $r_{q}$ with two adjacent robot nodes W and SE can leave the current node ((i): by Fig 6 (a), (ii): by Lemma 4 (iii): by Lemma 2 and (iv): by Lemma 5 .

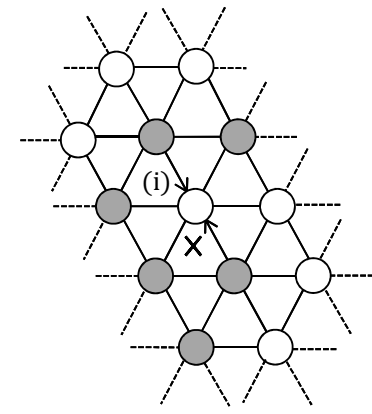

(a)

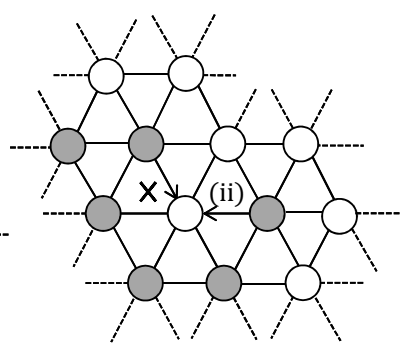

(b)
Fig. 33. Examples showing that a robot with two adjacent robot nodes W and SW cannot leave the current node ((i): assumption of Case 5-1, (ii): assumption of Case 5).

Case 5-1: robot $r_{q}$ moves to $S E$. In this case, a robot with two adjacent robot nodes SW and W cannot leave the current node because otherwise a collision may occur (Fig.33). Next, when considering a configuration of Fig. 34 , only robot $r_{i}$ with two adjacent robot nodes SE and SW can leave the current node. However, in a configuration of Fig. $35 r_{i}$ cannot move to $\mathrm{E}$ since a collision occurs. Hence, $r_{i}$ needs to move to $\mathrm{W}$. Then, a robot $r_{j}$ with two adjacent robot nodes $\mathrm{E}$ and NE cannot move to NW or SE because a collision occurs or the configuration become unconnected (Fig. 36. Finally, we consider the configuration of Fig. 37. In the figure, no robot can leave the current node and they cannot achieve gathering.

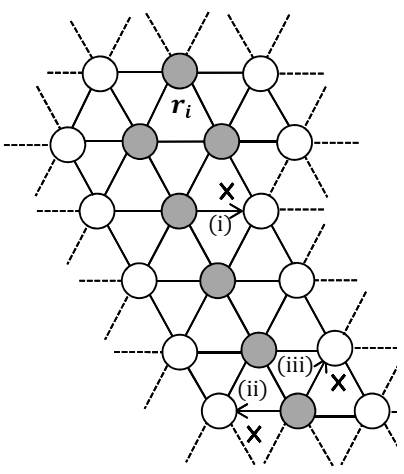

Fig. 34. A configuration such that only robot $r_{i}$ with two adjacent robot nodes SE and SW can leave the current node ((i): by Fig 31(d), (ii): by Lemma 2 (iii): by Lemma 5 .

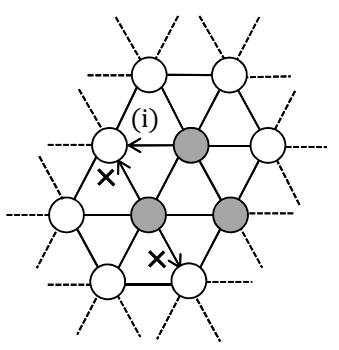

Fig. 36. An example showing that a robot with two adjacent robot nodes $\mathrm{E}$ and NE cannot leave the current node ((i): by Fig. 35.

Thus, a robot $r_{p}$ with two adjacent robot nodes SW and E cannot move to SE and it must stay at the current node.

Case 5-2: robot $r_{q}$ moves to $S W$. In this case, a robot with one adjacent robot node $\mathrm{E}$ cannot leave the current node because otherwise a collision may occur (Fig. 38). Then, when considering a configuration of Fig. 39. only robot $r_{i}$ with two adjacent robot nodes SW and $\mathrm{W}$ can leave the current node. However, $r_{i}$ cannot move to SE since a collision occurs in a configuration of Fig. 40. Hence, $r_{i}$ needs to move to NW. Then, robot $r_{j}$ with two adjacent robot nodes $\mathrm{E}$ and $\mathrm{SE}$ cannot leave the current node because a collision occurs or the configuration becomes unconnected (Fig.41). Next, we consider the configuration of Fig. 42 In the figure, only robot $r_{i}$ with three adjacent robot nodes SE, W, and NW can leave the current node and it needs to move to SW by the previous discussions. Then, a robot $r_{j}$ with four adjacent robot nodes 


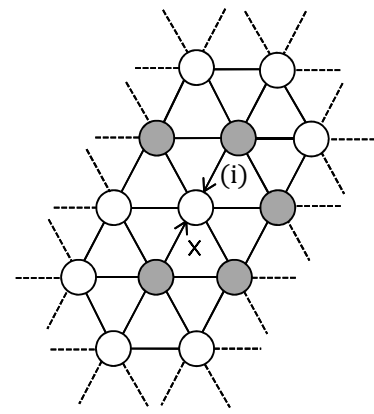

(a)

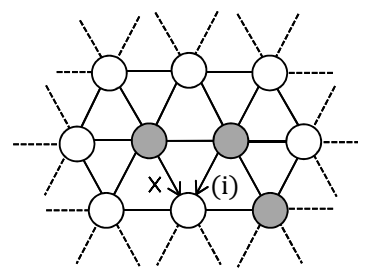

(b)
Fig. 38. Examples showing that a robot with two adjacent robot nodes $\mathrm{E}$ cannot leave the current node ((i): assumption of Case 5-2).

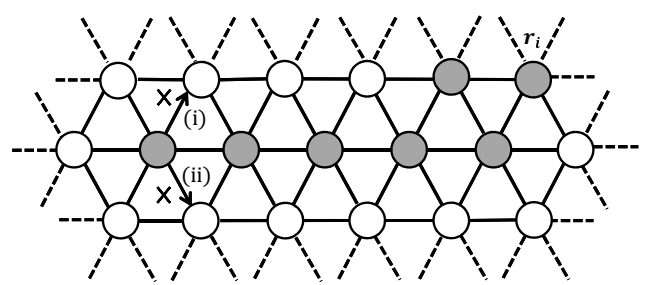

Fig. 39. A configuration such only robot $r_{i}$ with two adjacent robot nodes W and SW can leave the current node ((i): by Fig.38(a), (ii): by Fig.38(b)).

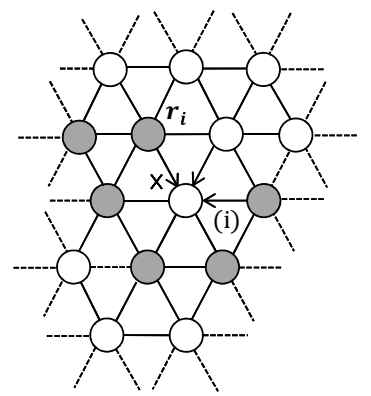

Fig. 40. An example showing that a robot with two adjacent robot nodes SW and W cannot move to SE ((i): assumption of Case 5).

E, SW, NW, and NE cannot leave the current node since a collision occurs in a configuration of Fig. 43 Thus, when considering the configuration of Fig.44, only robot $r_{i}$ with two adjacent robot nodes $\mathrm{W}$ and $\mathrm{NW}$ can leave the current node. However, $r_{i}$ cannot move to NE since a collision occurs in a configuration of Fig. 45 Thus, $r_{i}$ needs to move to SW. Then, a robot with one adjacent robot node $\mathrm{W}$ cannot leave the current node because otherwise a collision occurs in configurations of Fig.46. Finally, when considering the configuration of Fig.47. no robot can leave the current node and robots cannot achieve gathering, which is a contradiction. Thus, a robot $r_{q}$ with two adjacent robot nodes $\mathrm{W}$ and SE cannot move to SW. Therefore, we have the following lemma.
Fig. 41. An example showing that a robot with two adjacent robot nodes $\mathrm{E}$ and SE cannot leave the current node ((i): by Fig. 40.

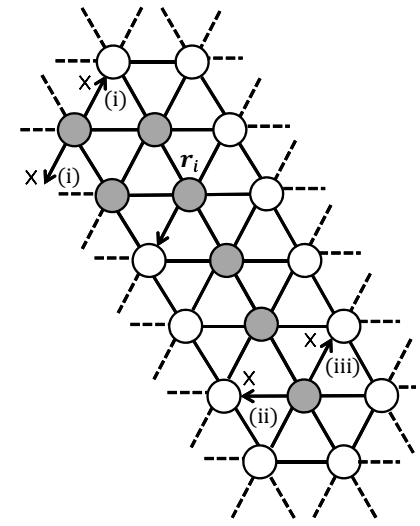

Fig. 42. A configuration such that only robot $r_{i}$ with three adjacent robot nodes SE, W, and NW can leave the current node ((i): by Fig. 41 (ii): by Lemma 2 (iii): by Lemma 5 .

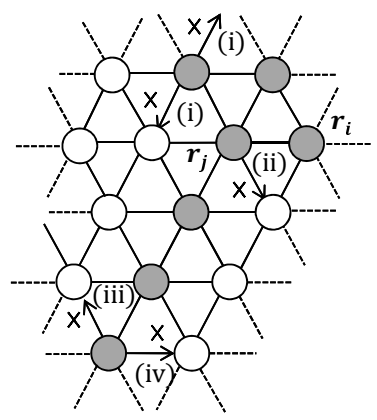

Fig. 44. A configuration such that only robot $r_{i}$ with two adjacent robot nodes $\mathrm{W}$ and NW can leave the current node ((i): by Fig.41 (ii): by Fig.43 (iii): by Fig.6 (a) (iv): by Lemma 4.

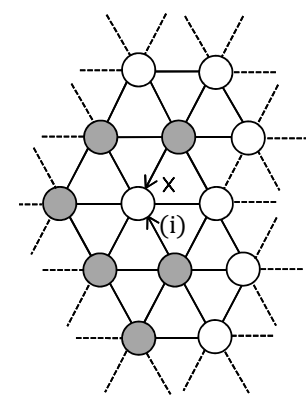

(a)

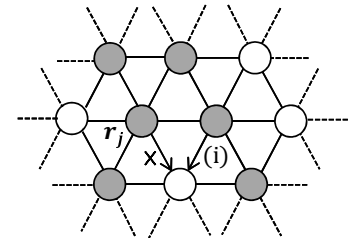

Fig. 43. An example showing that a robot $r_{j}$ with four adjacent robot nodes E, SW, NW, and NE cannot move to SE ((i): by Fig.42.

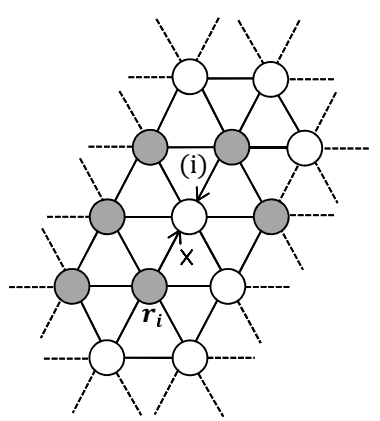

Fig. 45. An example showing that a robot $r_{i}$ with two adjacent robot nodes $\mathrm{W}$ and NW cannot move to NE ((i): assumption of Case 5-2).

\section{Fig. 46. Examples showing that a robot with one adjacent
cannot leave the current node ((i): by Fig. 40 (ii): by Fig. 45 .}

Lemma 6. A robot with one adjacent robot node SW cannot move to node $W$.

Thus, by Proposition 1.(a), Lemmas 1, 3, 4, and 5, robots cannot achieve gathering from a configuration of Fig. 4 (b) and 


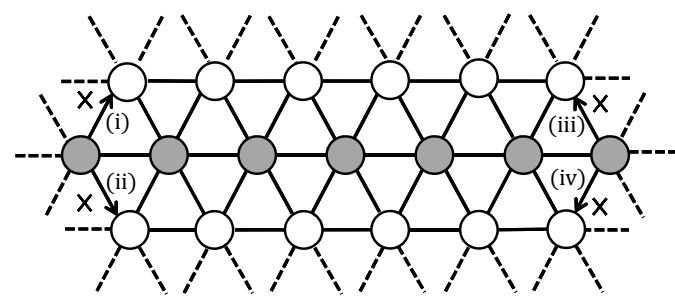

Fig. 47. An unsolvable configuration when a robot with two adjacent robot nodes W and SE moves to SW ((i): by Fig.38 (a), (ii): by Fig.38 (b), (iii): by Fig. 46(b), (iv): by Fig. 46 (a))

we have the theorem.

\section{ROBOTS WITH VISIBILITY RANGE 2}

In this section, for robots with visibility range 2, we propose a collision-free algorithm to solve the gathering problem from any connected initial configuration.

\section{A. Proposed algorithm}

The basic idea is that each robot firstly determines the base node that is the rightmost robot node within its visibility range and then it moves toward the base node to achieve gathering. First, we explain how to determine the base (or rightmost) robot. For explanation, in the following we assume that each robot $r_{i}$ recognizes that it is located at an origin and it assigns labels to each node within its visibility range like Fig. 48 In the figure, the first (resp., second) element of each label is called the $x$-element (resp., $y$-element) ${ }^{2}$ Then, $r_{i}$ determines the robot node with the largest $x$-element as the base node (possibly the robot node where $r_{i}$ itself stays). If several robot nodes have the largest $x$-element, $r_{i}$ does not determine the base node at that time and waits at the current node until the configuration changes. As exceptions, if node $(4,0)$ is an empty node and nodes $(3,1)$ and $(3,-1)$ are robot nodes, $r_{i}$ determines node $(4,0)$ as the base node to avoid the configuration such that no robot determines a base node and each robot waits at the current node. In addition, if robot nodes $(1,1)$ and $(1,-1)$ have the largest $x$-element among all the labels of robot nodes within $r_{i}$ 's visibility range, and $r_{i}$ moves to node $(2,0)$ so that it becomes a base. Examples are given in Fig. 49

Next, we explain how to achieve gathering based on the base node. Robots consider the base node as the rightmost node of a gathering-achieved configuration and they basically move east on a triangular grid with avoiding a collision and an unconnected configuration. Concretely, if the label of the base node from robot $r_{i}$ is $(2,-2),(3,-1),(4,0),(3,1)$, or $(2,2)$, it moves to one of adjacent nodes as indicated in Fig. 50 (a) using ordinal numbers in Fig.50 (b). That is, among the candidate nodes that $r_{i}$ may visit in the next cycle, $r_{i}$ moves to the empty adjacent node with the smallest ordinal number.

\footnotetext{
${ }^{2}$ Labels are assigned for explanation and they are a little different from the coordinate system. For example, the difference between labels $(0,0)$ and $(2,0)$ is 2 but the distance between node $(0,0)$ and node $(2,0)$ is 1 .
}

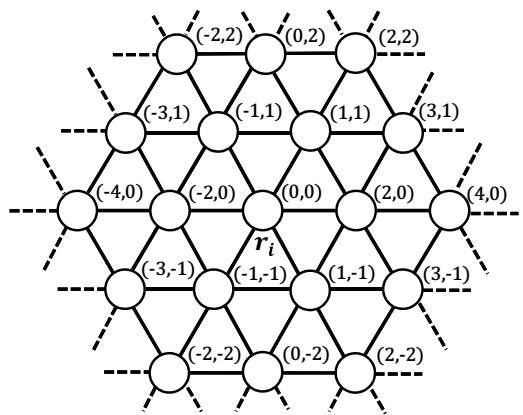

Fig. 48. Assignment of labels.

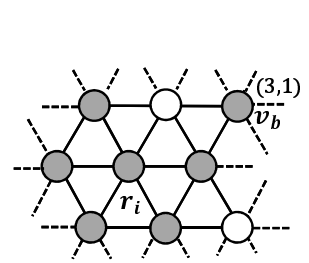

(a)

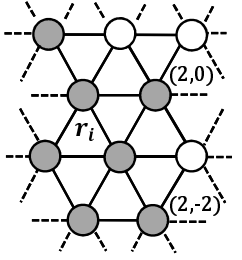

(b)

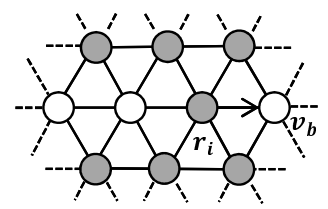

(c)
Fig. 49. Examples of how to determine the base nodes ((a): node $v_{b}$ is the base node, (b): $r_{i}$ does not determine the base node, (c): $r_{i}$ determines $v_{b}$ as the base node and moves there)

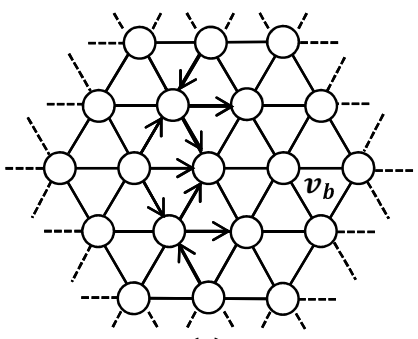

(a)

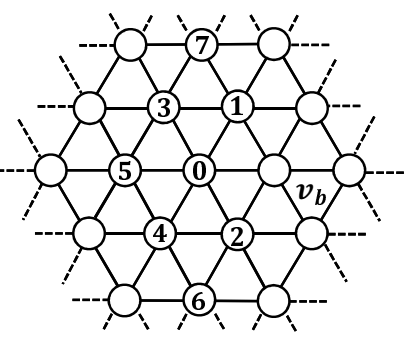

(b)
Fig. 50. Movement rules ((a): candidate nodes to visit, (b): ordinal numbers).

If several robots try to move to the same node $v_{j}$, the robot staying at the node with the largest ordinal number moves to $v_{j}$. If all the candidate nodes are robot nodes, $r_{i}$ stays at the current node. For example, in Fig.51, robots $r_{i}$ and $r_{j}$ consider the common node $v_{b}$ as the base node, $r_{i}$ (resp., $r_{j}$ ) has two candidate nodes $v_{n}$ and $v_{\ell}$ (resp., $v_{m}$ and $v_{\ell}$ ) to visit, $v_{n}$ (resp., $v_{m}$ ) is a robot node, and hence it tries to visit $v_{\ell}$ (resp., $v_{\ell}$ ). In this case, since the ordinal number 4 of the node where $r_{i}$ stays is larger than the ordinal number 3 of the node where $r_{j}$ stays, $r_{i}$ moves to $v_{\ell}$ and $r_{j}$ stays at the current node. If two robots consider the common node as the base node like the above example, they can share the common ordinal numbers and can avoid a collision or an unconnected configuration. However, it is possible that some two robots consider different robot nodes as their base nodes due to their limited visibility range, which may cause a collision or an unconnected configuration. For example, in Fig.52, robot $r_{i}$ considers $v_{b}^{\prime}$ as the base node but $r_{j}$ considers $v_{b}$ as the base node, and they try to move to the same node $v_{\ell}$ according to 


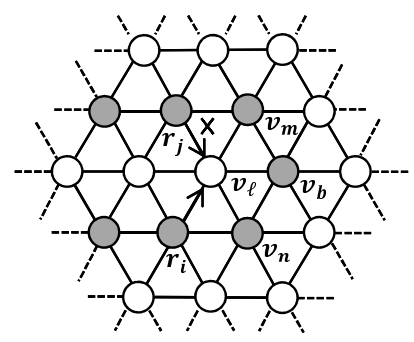

Fig. 51. An example to avoid a collision using ordinal numbers.

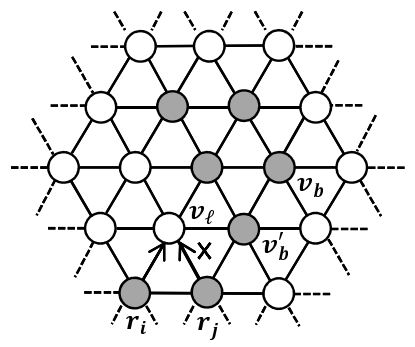

Fig. 52. An example to avoid a collision using $x$-elements.

the movement rule. In this case, the robot with the smaller $x$-element of the node label moves to the node and the other robot stays at the current node. Hence, in Fig. 52, $r_{i}$ moves to $v_{\ell}$ and $r_{j}$ stays at the current node. Moreover, only with the movement rule in Fig. 50 no robot leaves the current node in the configuration in Fig. 53. In this case, as a special behavior, if the label of the base node from robot $r_{i}$ is $(3,1)$, nodes $(1,1)$, $(2,0)$, and $(1,-1)$ are robot nodes, and node $(-1,1)$ is an empty node, $r_{i}$ moves to the northwest adjacent node $(-1,1)$ so that robot $r_{j}$ staying at $r_{i}$ 's southeast adjacent node $(1,-1)$ can move to the node where $r_{i}$ is currently staying. When robots reach a configuration such that no robot leaves the current node, the configuration is one solution of the gathering problem.

An example of the algorithm execution is given in Fig.54. From (a) to (b), since $r_{2}$ 's northeast and southeast adjacent nodes are robot nodes and no other robot node has larger $x$ element than that of the node where $r_{2}$ stays, it moves to east adjacent node $v_{b}$. From (b) to (c), robots $r_{0}$ and $r_{3}$ consider $v_{b}$ as the common base node and they try to move to the empty node $v_{k}$ with the smallest ordinal number among candidate empty nodes. In this case, since the ordinal number of the node where $r_{3}$ stays is larger than that of the node where $r_{0}$ stays, $r_{3}$ moves to $v_{k}$ and $r_{0}$ stays at the current node. From (c) to (d), $r_{5}$ (resp., $r_{6}$ ) considers $v_{b}^{\prime}$ (resp., $v_{b}$ ) as the base node and they try to move to node $v_{\ell}$. In this case, since the $x$-element of the node that where $r_{5}$ stays is smaller than that of the node where $r_{6}$ stays, $r_{5}$ moves to $v_{\ell}$ and $r_{6}$ stays at the current node. From (d) to (e), as a special behavior, robot $r_{5}$ moves to the northwest adjacent robot node so that $r_{6}$ can move to the node where $r_{5}$ is currently staying. From (e) to (f), robot $r_{6}$ considers $v_{b}$ as the base node and it moves to the northeast adjacent node. Then, robots achieve gathering.

The pseudocode of the proposed algorithm is described in Algorithm 1 In the following, we explain several robot behaviors that avoid a collision or an unconnected configuration. The behavior of robot $r_{i}$ for the case that, the label of the base node is $(2,0)$ but the node is an empty node, is described in lines $1-$ 3 . In this case, $r_{i}$ tries to move to node $(2,0)$. However, if $r_{i}$ 's west adjacent node $(-2,0)$ is a robot node and $r_{i}$ moves to the base node $(2,0)$, the configuration may become unconnected (Fig.55 (a)). Hence, in this case $r_{i}$ moves to node $(2,0)$ when $r_{i}$ 's northwest or southwest adjacent node is also a robot node (Fig.55 (b)).

The behavior of robot $r_{i}$ for the case that the label of the base node is $(4,0)$ is described in lines $5-9$. In this case, if node $(2,0)$ is an empty node, $r_{i}$ tries to move to the node. However, if $r_{i}$ 's southwest adjacent node $(-1,-1)$ is a robot node and $r_{i}$ moves to node $(2,0)$, the configuration may become unconnected (Fig.56 (a)). Hence, in this case $r_{i}$ moves to node $(2,0)$ when its southeast adjacent node $(1,-1)$ is also a robot node (Fig.56(b)).

The behavior of robot $r_{i}$ for the case that the label of the base node is $(3,-1)$ is described in lines $11-15$. In this case, if nodes $(2,0),(1,-1)$ and $(1,1)$ are robot nodes and node $(-1,-1)$ is an empty node, $r_{i}$ tries to move to its southwest adjacent node $(-1,-1)$ so that the robot staying at node $(1,1)$ could move to node $(0,0)$ where $r_{i}$ is currently staying. However, due to the limited visibility range, it is possible that $r_{i}$ and some robot $r_{j}$ consider different nodes as base nodes, $r_{j}$ staying at node $(-2,0)$ or $(-2,-2)$ tries to move to node $(-1,-1)$, and a collision occurs (Fig. 57 (a), (b)). Hence, in this case $r_{i}$ moves to node $(-1,-1)$ when nodes $(-2,0),(-2,-2)$, and $(-1,1)$ are empty nodes (Fig.57 (c)). In addition, if node $(1,-1)$ is an empty node, $r_{i}$ tries to move to the node. Then, it is possible that $r_{i}$ and some robot $r_{j}$ consider different nodes are base nodes, $r_{j}$ staying at node $(-1,1)$ tries to move to node $(-2,0)$, and the configuration become unconnected (Fig. 57(d)). Hence, in this case $r_{i}$ moves to node $(1,-1)$ when node $(0,-2)$ is an empty node.

The behavior of robot $r_{i}$ for the case that the label of the base node is $(2,2)$ is described in lines $27-29$. In this case, if node $(1,1)$ is a robot node and node $(-1,1)$ is an empty node, it tries to move to node $(-1,1)$. However, due to the limited visibility range, it is possible that $r_{i}$ and some robot $r_{j}$ consider different nodes are base nodes, $r_{j}$ staying at node ($2,0)$ tries to move to node $(-1,1)$, and a collision occurs (Fig. 58 (a)), or $r_{j}$ staying at node $(-1,-1)$ does not leave the current node and the configuration becomes unconnected (Fig.58(b)). Hence, in this case $r_{i}$ moves to node $(-1,-1)$ when nodes $(-2,0)$ and $(-1,-1)$ are empty nodes (Fig. $58(\mathrm{c})$ ).

Although there still exist several robot behaviors that avoid a collision or an unconnected configuration, we omit the detail.

\section{B. Correctness}

The correctness of the proposed algorithm has been evaluated by computer simulations. By the simulations, we confirmed that robots which execute the proposed algorithm can achieve gathering from all possible connected initial configurations (3652 patterns in total) in the fully synchronous (FSYNC) model. Thus, we have the following theorem.

Theorem 2. For robots with visibility range 2, the proposed algorithm solves the gathering problem from any connected initial configuration in the FSYNC model.

\section{CONCLUSION}

In this paper, we considered the gathering problem of seven autonomous mobile robots on triangular grid graphs. First, for robots with visibility range 1 , we showed that no collision-free algorithm exists for the gathering problem. Next, for robots with visibility range 2 , we proposed a collision-free algorithm 


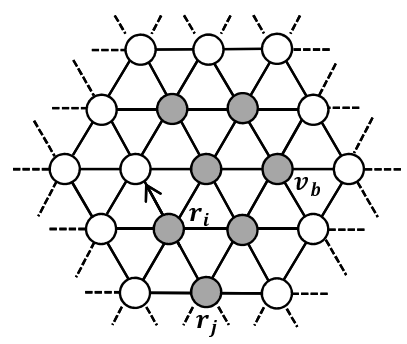

Fig. 53. An example to avoid a standstill.

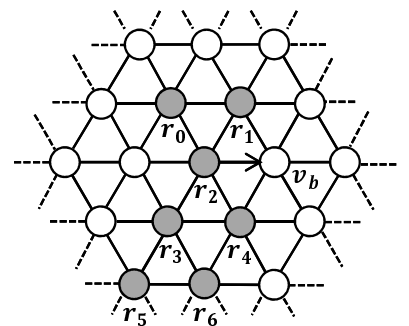

(a)

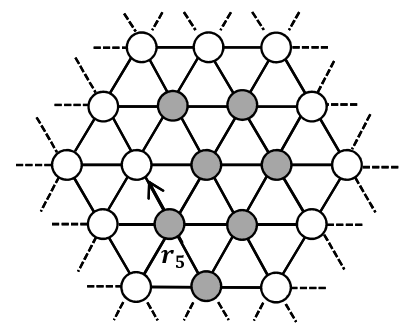

(d)

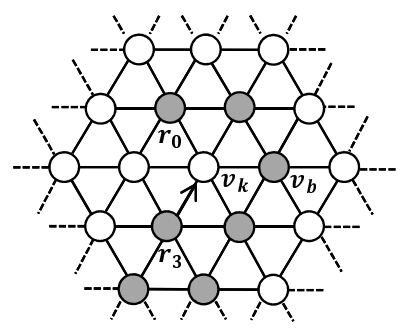

(b)

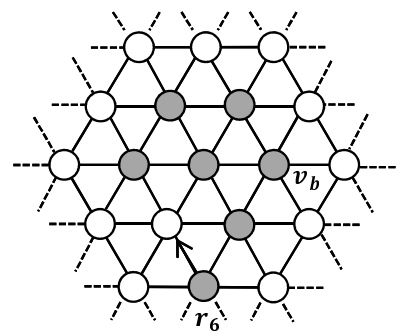

(e)

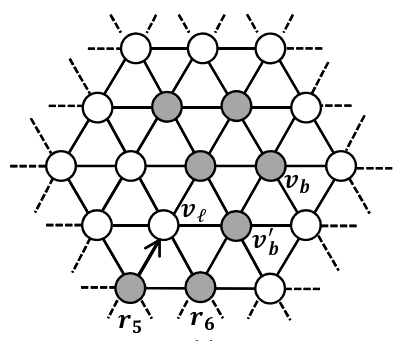

(c)

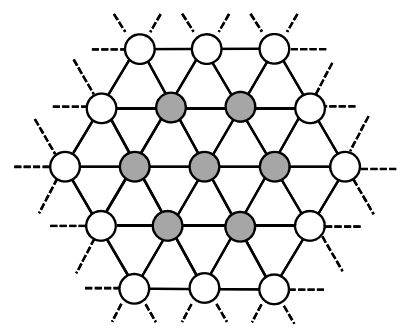

(f)

Fig. 54. An example of the algorithm execution.

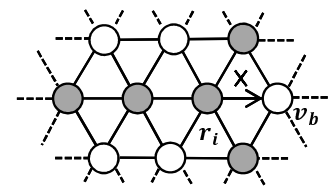

(a)

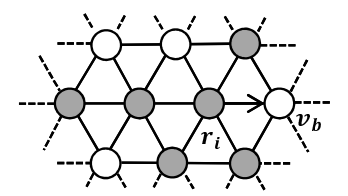

(b)
Fig. 55. Behavior of robot $r_{i}$ when the label of the base node $v_{b}$ is $(2,0)$ but the node is an empty node.

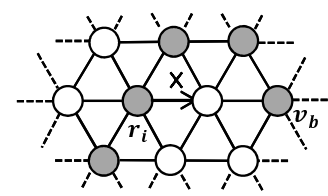

(a)

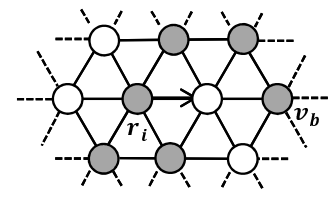

(b)
Fig. 56. Behavior of robot $r_{i}$ when the label of the base node $v_{b}$ is $(4,0)$.

to solve the problem from any connected initial configuration. This algorithm is optimal in terms of visibility range.

There are four possible future works as follows. First, we will complete a theoretical proof of correctness for the proposed algorithm in Section IV. Second, we will consider the relaxed version of connected initial configuration such that the visibility relationship among robots constitutes one connected graph. Third, we will consider gathering for different number of robots. Lastly, we consider other problems such as the pattern formation problem for autonomous mobile robots on triangular grids.

\section{ACKNOWLEDGEMENT}

This work was partially supported by JSPS KAKENHI Grant Number 18K18029, 18K18031, 19K11823, 20H04140, and 20KK0232; the Hibi Science Foundation; and Foundation of Public Interest of Tatematsu.

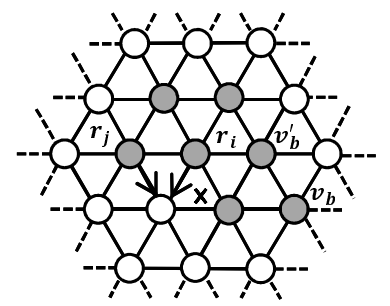

(a)

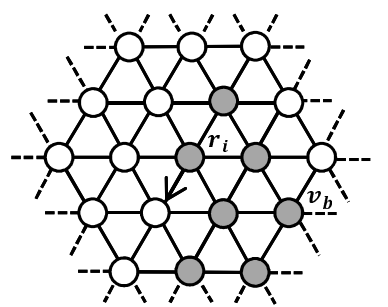

(c)

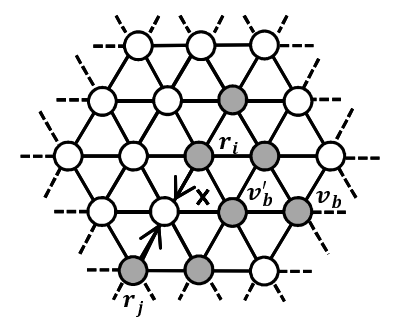

(b)

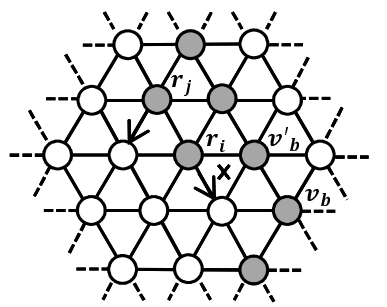

(d)
Fig. 57. Behavior of robot $r_{i}$ when the label of the base node $v_{b}$ is $(3,-1)$ $\left(v_{b}^{\prime}\right.$ : the base node for robot $r_{j}$ ).

\section{REFERENCES}

[1] I. Suzuki and M. Yamashita. Distributed anonymous mobile robots: Formation of geometric patterns. SIAM Journal on Computing, 28(4):13471363, 1999.

[2] P. Courtieu, L. Rieg, S. Tixeuil, and X. Urbain. Impossibility of gathering, a certification. Information Processing Letters, 115(3):447452, 2015.

[3] Y. Dieudonné and F. Petit. Self-stabilizing gathering with strong multiplicity detection. Theoretical Computer Science, 428:47-57, 2012.

[4] G. D'Angelo, G. Di Stefano, R. Klasing, and A. Navarra. Gathering of robots on anonymous grids and trees without multiplicity detection. Theoretical Computer Science, 610:158-168, 2016.

[5] J. Castenow, M. Fischer, J. Harbig, D. Jung, and FM. auf der Heide. Gathering anonymous, oblivious robots on a grid. Theoretical Computer Science, 815:289-309, 2020. 


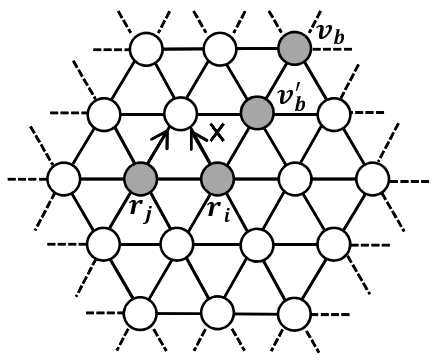

(a)

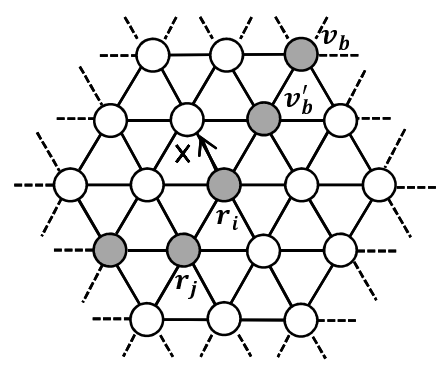

(b)

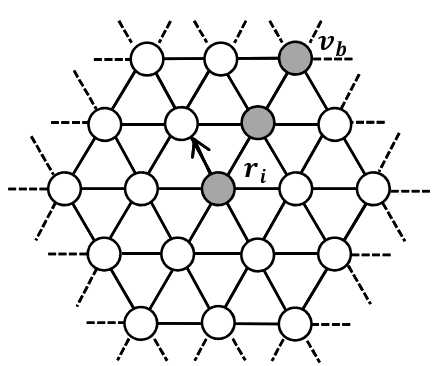

(c)

Fig. 58. Behavior of robot $r_{i}$ when the label of the base node $v_{b}$ is $(2,2)\left(v_{b}^{\prime}\right.$ : the base node for robot $\left.r_{j}\right)$.

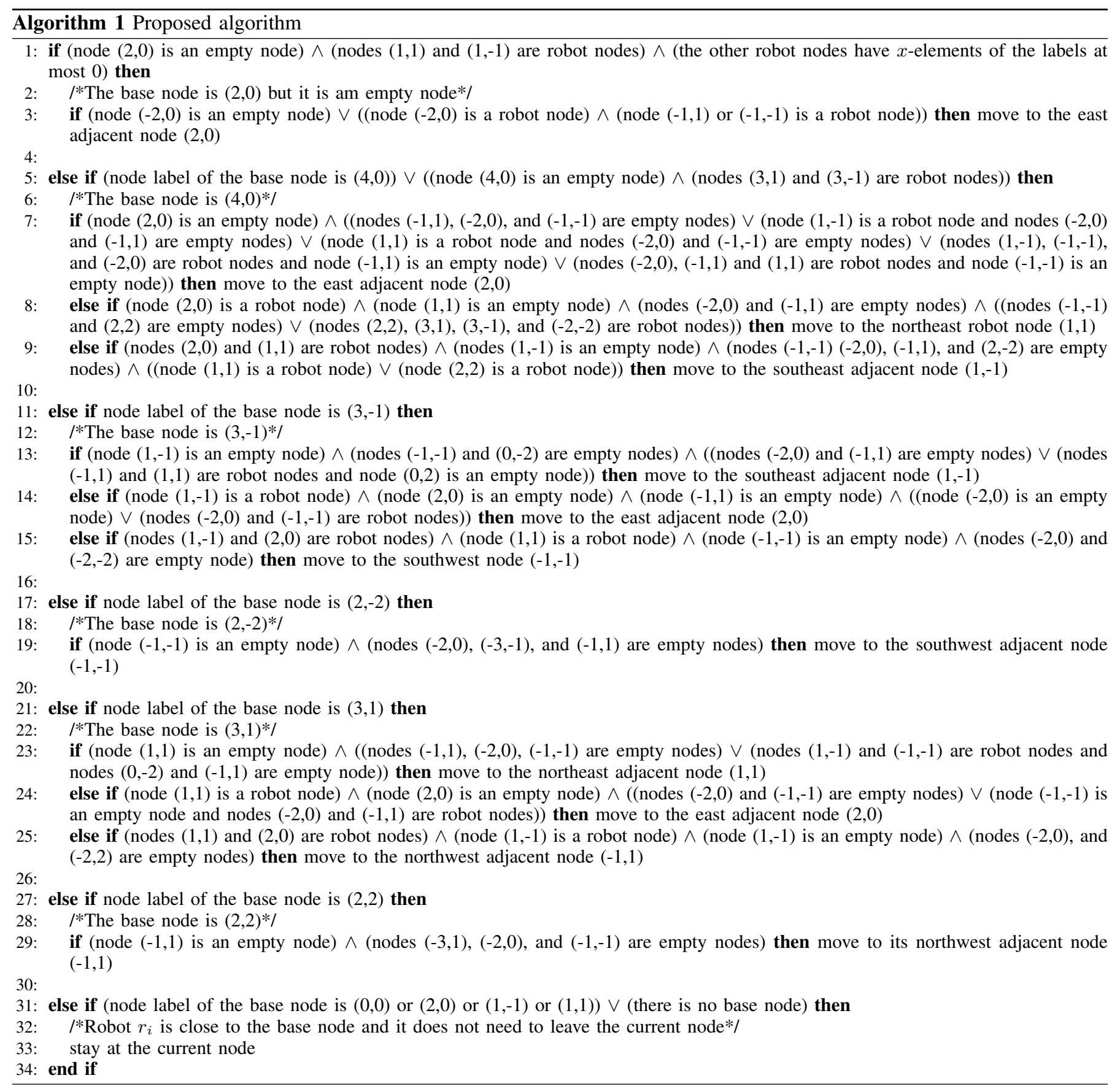


[6] R. Klasing, A. Kosowski, and A. Navarra. Taking advantage of symmetries: Gathering of many asynchronous oblivious robots on a ring. Theoretical Computer Science, 411(34-36):3235-3246, 2010.

[7] R. Klasing, E. Markou, and A. Pelc. Gathering asynchronous oblivious mobile robots in a ring. Theoretical Computer Science, 390(1):27-39, 2008.

[8] G. D'Angelo, G. Di Stefano, and A. Navarra. Gathering on rings under the look-compute-move model. Distributed Computing, 27(4):255-285, 2014.

[9] G. Di Stefano and A. Navarra. Optimal gathering of oblivious robots in anonymous graphs and its application on trees and rings. Distributed Computing, 30(2):75-86, 2017.

[10] J. Czyzowicz, L. Gasieniec, and A. Pelc. Gathering few fat mobile robots in the plane. Theoretical Computer Science, 410(6-7):481-499, 2009.

[11] C. Agathangelou, C. Georgiou, and M. Mavronicolas. A distributed algorithm for gathering many fat mobile robots in the plane. Proc. PODC, pages 250-259, 2013 .

[12] Y. Ito, Y. Katayama, and K. Wada. A gathering problem for nobile fat robots in a grid without the global coordinate system. Technical Report of IEICE (COMP2014-10), 114:53-59, 2014.

[13] Z. Derakhshandeh, S. Dolev, R. Gmyr, AW. Richa, C. Scheideler, and T. Strothmann. Brief announcement: amoebot-a new model for programmable matter. In Proc. SPAA, pages 220-222. ACM, 2014.

[14] J. J Daymude, R. Gmyr, A. W Richa, C. Scheideler, and T. Strothmann. Improved leader election for self-organizing programmable matter. ALGOSENSORS, pages 127-140, 2017.

[15] S. Cannon, JJ. Daymude, D. Randall, and AW. Richa. A markov chain algorithm for compression in self-organizing particle systems. Proc. PODC, pages 279-288, 2016.

[16] G. A. Di Luna, P. Flocchini, N. Santoro, G. Viglietta, and Y. Yamauchi. Shape formation by programmable particles. Distributed Computing, 33(1):69-101, 2020.

[17] Z. Derakhshandeh, R. Gmyr, AW. Richa, C. Scheideler, and T. Strothmann. Universal shape formation for programmable matter. Proc. PODC, pages 289-299, 2016. 